\title{
Modelling and Simulating the Efficiency and Elasticity of Gearboxes
}

\author{
F.L.J. van der Linden P.H. Vazques de Souza Silva \\ German Aerospace Center (DLR) \\ Institute of Robotics and Mechatronics, Oberpfaffenhofen, Germany
}

\begin{abstract}
Two elastic gearbox models with friction losses are presented; a 1-Degree Of Freedom (DOF) and a 3DOF model.

The presented models have advantages over the existing lossy gear model [7] of the Modelica Standard Library when gear vibrations are of interest. Care has been taken that also in the case of gear locking, the elasticity effects are treated adequately.

In addition to external excitations, it is now also possible to model internal excitations of the gearbox caused by the varying stiffness and/ or damping. This varying stiffness can be specified by the user for each gear wheel. This feature can for instance be used to model tooth interaction or broken gears.

Furthermore, the 3-DOF elastic model can simulate the elasticity of the support bearings in the load direction, which is impossible in the standard lossy gear model.

Keywords: Elastic Gearbox, Efficiency, Gearbox
\end{abstract}

\section{Introduction}

Gearbox vibrations and losses can affect the performance of a mechanical system as a whole. For example in wind turbines, vibrations of gearboxes often cause undesired behaviour or even fatigue failures. Moreover, in other applications elastic effects of the gearbox can influence the performance of the system, especially in low weight - high gear ratio applications, such as lightweight robots and aircraft applications. In this article it is presented how the lossy gear model from the Modelica Standard Library Modelica.Mechanics.Rotational addressed by Pelchen et al. [7] is extended to a full elastic model, without losing the symmetry of the model. Two models have been developed; a 1-DOF model and a 3-DOF model.

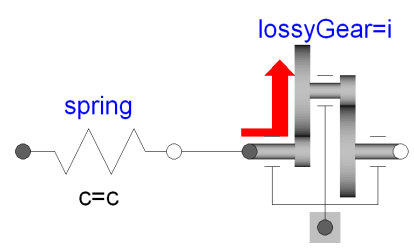

(a) asymmetric elastic gear

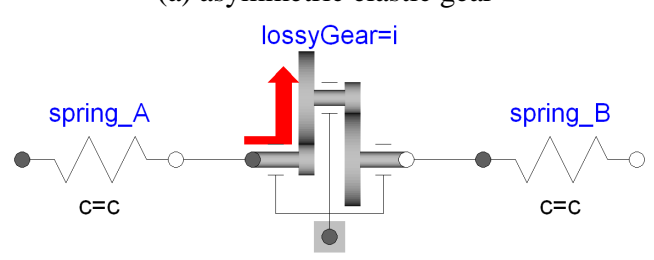

(b) symmetric elastic gear

Figure 1: Lossy gear from the Modelica Standard Library, extended with springs to create an elastic gear model

\section{Overview of Available Models}

The lossy gear model can simulate the efficiency of the gearbox depending on load direction and speed as well as stick slip effects. Bearing friction effects are also included in the model. The standard lossy gear model is a rigid model. When elastic effects are needed for correct modelling, constructions as in Figure 1 can be made to simulate elasticity. Figure 1a yields in the case of a locked gear a non-symmetric model since the elasticity is lumped on one side. In many cases this leads to non-realistic model behaviour.

The model shown in Figure 1b has problems to be simulated at all. See Appendix A for an in-depth analysis of this problem.

Sing and Houser [8] have developed an elastic gear model that can simulate torsional as well as transverse vibrations. Mesh- and bearing losses, however, are not taken into account. Howard et al. [1] have been working with FEM models. These models are highly complex and the simulation times are high. Moreover the geometry and material properties have to be known, 
which is usually not the case.

In this report, the work of Pelchen et al. [7] on the standard lossy gear model is combined with the work of Sing and Houser [8]. The goal of this paper is to present a low order symmetric model that can simulate mesh- and bearing losses as well as an elastic contact. The parameters needed for the model can either be found in vendor catalogues or can be measured without spending much time and resources.

\section{The Elastic Lossy Gear Model}

Two elastic gear models are developed; a torsional elastic model (Figure 2a, 1-DOF) and a translationaltorsional model (Figure 2b, 3-DOF). With the first model the effect of elastic teeth in a gearbox can be simulated. The second model adds elasticity of the bearings in the direction of the gear load.

A schematical overview of the power flow through the lossy gear model is shown in Figure 3. The symbols and their description are listed in Table 1.

The mesh is modelled using a spring and a damper on each side of the contact position $y_{\text {mesh }}$. The mesh forces $F_{m A}$ and $F_{m B}$ are related to the mesh torques $\tau_{m A}$ and $\tau_{m B}$ as:

$$
\begin{aligned}
& F_{m A}=\frac{\tau_{m A}}{r_{A}}=\frac{\tau_{g A}-\tau_{\text {loss }}}{r_{A}} \\
& F_{m B}=-\frac{\tau_{m B}}{r_{B}}=-\frac{\tau_{g B}-\tau_{\text {loss }}}{r_{B}}
\end{aligned}
$$

And the resulting driving forces are (These are the forces left after all friction losses):

$$
F_{g A}=\frac{\tau_{g A}}{r_{A}}, \quad F_{g B}=-\frac{\tau_{g B}}{r_{B}}
$$

The spring forces $F_{m A}$ and $F_{m B}$ in the load direction are for the 1-DOF model (see Figure 2a):

$$
\begin{aligned}
& F_{m A}=k_{h A}\left(y_{m e s h}-r_{A} \theta_{A}\right)+c_{h A}\left(v_{m e s h}-r_{A} \dot{\theta}_{A}\right) \\
& F_{m B}=k_{h B}\left(r_{B} \theta_{B}-y_{m e s h}\right)+c_{h B}\left(r_{B} \dot{\theta}_{B}-v_{m e s h}\right)
\end{aligned}
$$

For the 3-DOF model (see Figure 2a) it results:

$$
\begin{aligned}
F_{m A}= & k_{h A}\left(y_{m e s h}-\left(r_{A} \theta_{A}+y_{A}\right)\right)+ \\
& c_{h A}\left(v_{m e s h}-\left(r_{A} \dot{\theta}_{A}+\dot{y}_{A}\right)\right) \\
F_{m B}= & k_{h B}\left(\left(r_{B} \theta_{B}+y_{B}\right)-y_{m e s h}\right)+ \\
& c_{h B}\left(\left(r_{B} \dot{\theta}_{B}+\dot{y}_{A}\right)-v_{\text {mesh }}\right)
\end{aligned}
$$

\begin{tabular}{|c|c|}
\hline Symbol & Description \\
\hline$\overline{m_{\mathscr{J}}}$ & "Mass wheel $\mathscr{I}$ \\
\hline$r_{\mathscr{I}}$ & Radius of wheel $\mathscr{I}$ \\
\hline$I_{g \mathscr{I}}$ & Mass moment of inertia of wheel $\mathscr{I}$ \\
\hline$k_{h \mathscr{I}}$ & Gear contact spring constant wheel $\mathscr{I}$ \\
\hline$k_{h}$ & Total gear contact spring constant \\
\hline$k_{b \mathscr{I}}$ & Bearing stiffness wheel $\mathscr{I}$ \\
\hline$\Delta k_{h \mathscr{I}}$ & normalized stiffness profile \\
\hline$k_{h, \text { base }}$ & $k_{h}=k_{h, \text { base }} \Delta k_{h \mathscr{I}}$ \\
\hline$c_{h \mathscr{I}}$ & $\begin{array}{l}\text { Gear contact damping constant } \\
\text { wheel } \mathscr{I}\end{array}$ \\
\hline$c_{h}$ & Total gear contact damping constant \\
\hline$c_{b \mathscr{I}}$ & Bearing damping wheel $\mathscr{I}$ \\
\hline$\Delta c_{h \mathscr{I}}$ & normalized damping profile \\
\hline$c_{h, \text { base }}$ & $c_{h}=c_{h, \text { base }} \Delta c_{h \mathscr{I}}$ \\
\hline$y_{\mathscr{I}}$ & Displacement of wheel $\mathscr{I}$ \\
\hline$y_{m \mathscr{I}}$ & $y_{\mathscr{I}}+r_{\mathscr{I}} \theta_{\mathscr{I}}$ \\
\hline$y_{\text {mesh }}$ & $\begin{array}{l}\text { Displacement in load direction of } \\
\text { the contact point }\end{array}$ \\
\hline$v_{m e s h}$ & $\dot{y}_{m e s h}$ \\
\hline$\theta_{\mathscr{I}}$ & Angular position of wheel $\mathscr{I}$ \\
\hline$\tau_{\mathscr{I}}$ & Input torque on shaft $\mathscr{I}$ \\
\hline$\tau_{m \mathscr{I}}$ & Mesh torque of shaft $\mathscr{I}$ \\
\hline$\tau_{g \mathscr{I}}$ & Resulting driving toque of shaft $\mathscr{I}$ \\
\hline$\tau_{\text {loss } \mathscr{I}}$ & Mesh loss torque on shaft $\mathscr{I}$ \\
\hline$\tau_{b f \mathscr{I}}$ & Bearing friction torque on shaft $\mathscr{I}$ \\
\hline$\tau_{\text {loss,max } \mathscr{I}}$ & Maximal loss torque of gear $\mathscr{I}$ \\
\hline$\tau_{\text {loss }, \min \mathscr{I}}$ & Minimal loss torque of gear $\mathscr{I}$ \\
\hline$F_{m \mathscr{I}}$ & Mesh force of gear wheel $\mathscr{I}$ \\
\hline$F_{g \mathscr{I}}$ & Resulting driving force of gear $\mathscr{I}$ \\
\hline$F_{\text {loss } \mathscr{I}}$ & Mesh loss force on shaft $\mathscr{I}: \frac{\tau_{\mathscr{g} \mathscr{I}}}{r_{\mathscr{G}}}$ \\
\hline$F_{b \mathscr{I}}$ & Bearing force wheel $\mathscr{I}$ \\
\hline$\Delta F_{h}$ & $\begin{array}{l}\text { Force difference over the mesh } \\
\qquad \Delta F_{g}=F_{g B}-F_{g A}\end{array}$ \\
\hline$F_{\text {loss }, \max \mathscr{I}}$ & Maximal loss force of gear $\mathscr{I}$ \\
\hline$F_{l o s s, \min \mathscr{I}}$ & Minimal loss firce of gear $\mathscr{I}$ \\
\hline$P_{\text {loss }}$ & Power loss of the gear mesh \\
\hline$P_{\text {loss } \mathscr{I}}$ & Power loss of the gear $\mathscr{I}$ \\
\hline$P_{m \mathscr{I}}$ & Power flow into mesh $\mathscr{I}$ \\
\hline
\end{tabular}

Since in a gear mesh the gear moduli of the meshing teeth have to be equal, the following assumption is postulated:
Table 1: List of symbols. 


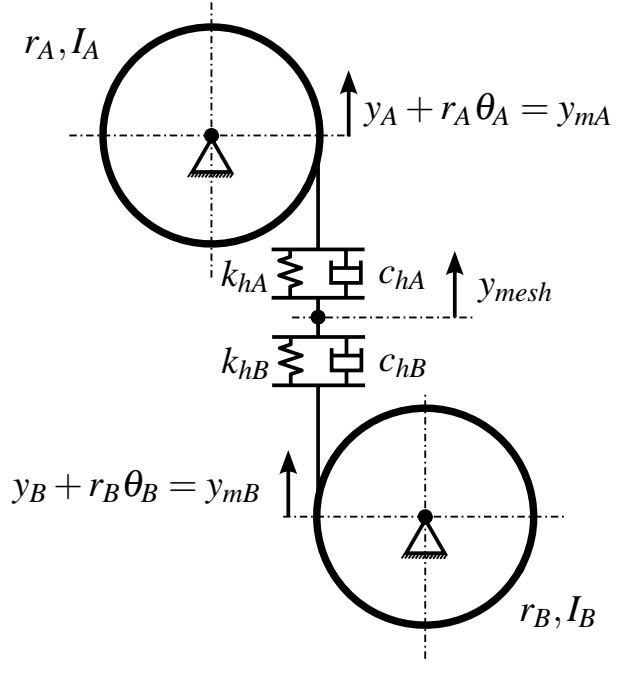

(a) 1-DOF Elastic Gear

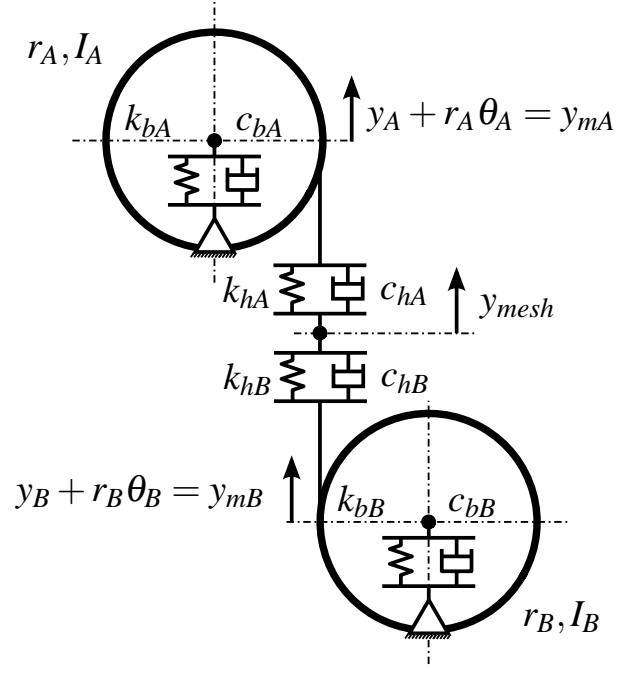

(b) 3-DOF Elastic Gear

Figure 2: The One Degree and the Three Degrees of Freedom Elastic Gear Models

Assumption 1 The mesh stiffness and the mesh damping are equal on both gear wheels

This leads to:

$$
\begin{aligned}
& k_{h}=2 k_{h A}=2 k_{h b} \\
& c_{h}=2 c_{h A}=2 c_{h b}
\end{aligned}
$$

The bearing forces are given by:

$$
\begin{aligned}
& F_{b A}=-\left(k_{b A} y_{A}+c_{b A} \dot{y}_{A}\right) \\
& F_{b B}=-\left(k_{b B} y_{B}+c_{b B} \dot{y}_{B}\right)
\end{aligned}
$$

In Figure 3 the torques and forces on both gear wheels are shown. Using the sign conventions from this figure, the rotational and translational equations of mo-

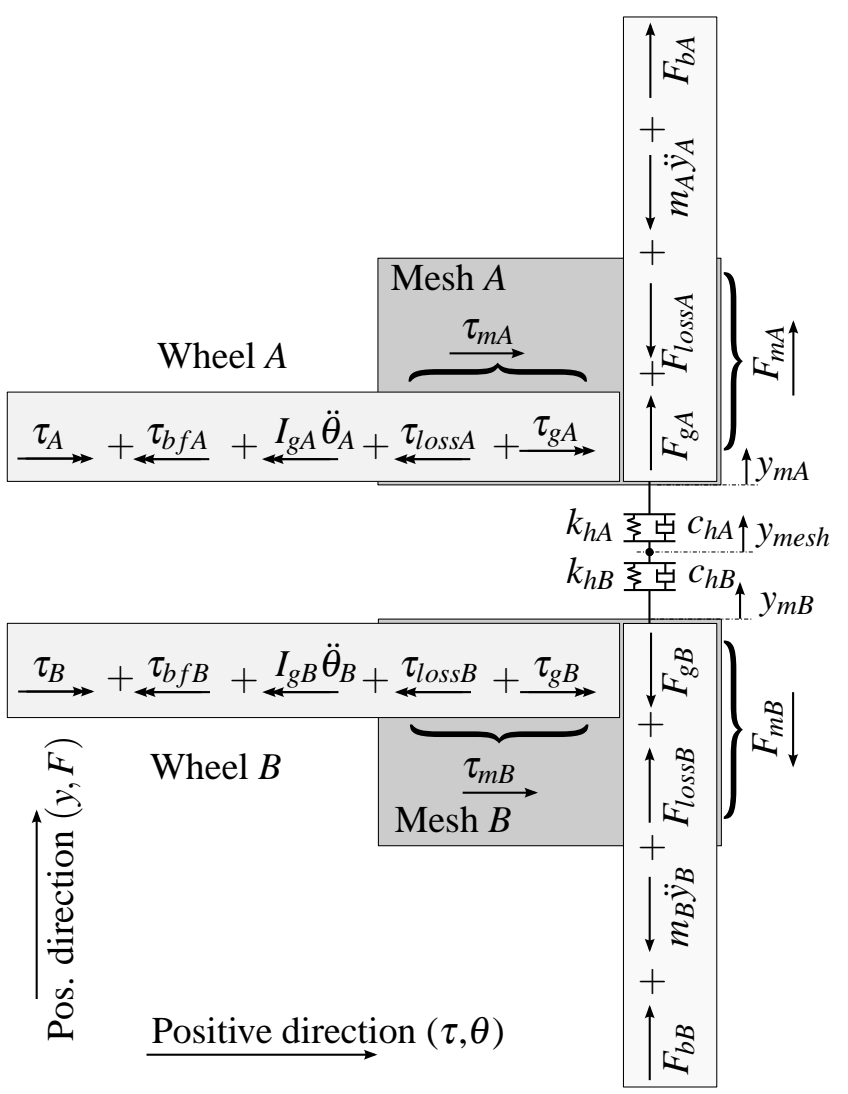

Figure 3: Forces and moments on the gearbox. The Torque/ Force convention is also shown.

tion can be obtained.

$$
\begin{aligned}
\tau_{A}-\tau_{b f A}-I_{g A} \ddot{\theta}_{A}-\tau_{\text {loss } A}+\tau_{g A} & =0 \\
\tau_{B}-\tau_{b f B}-I_{g B} \ddot{\theta}_{B}-\tau_{\text {loss } B}+\tau_{g B} & =0 \\
F_{b A}-m_{A} \ddot{y}_{A}-F_{\text {loss }}+F_{g A} & =0 \\
F_{b B}-m_{B} \ddot{y}_{B}+F_{\text {loss } B}-F_{g B} & =0
\end{aligned}
$$

Equations 14 and 15 reduce for the 1-DOF model to:

$$
\begin{aligned}
F_{\text {loss } A}-F_{g A} & =0 \\
-F_{\text {loss } B}+F_{g B} & =0
\end{aligned}
$$

For a moving gear (not stuck) this coupling equation between hull $A$ and $B$ is defined by the resultant drive forces:

$$
F_{g A}=F_{g B}
$$

In stuck mode though, hull $A$ and $B$ are uncoupled. Since the gear is stuck, the constraint equation is:

$$
v_{\text {mesh }}=0
$$

\subsection{Gear Mesh Losses}

For the gear mesh efficiency it is important to identify the power in- and outflows of the gear mesh. These 


\begin{tabular}{c||c|c} 
& $F_{h Y}>0$ & $F_{h Y}<0$ \\
\hline \hline$v_{\text {mesh }}>0$ & Quadrant 1 & Quadrant 2 \\
\hline$v_{\text {mesh }}<0$ & Quadrant 4 & Quadrant 3
\end{tabular}

Table 2: Gear operational modes and quadrants. The power flow in quadrant 1 and 3 is from Gearwheel $A$ to Gearwheel $B$, in quadrant 2 and 4 from Gearwheel $B$ to Gearwheel $A$

powers can be obtained by rearranging Equation 12 and 13 and multiplying them by the rotational velocity. The power that flows into the gear hull is therefore:

$$
\begin{aligned}
P_{m, A} & =\left(\tau_{A}-\tau_{b f A}-I_{g A} \ddot{\theta}_{A}\right) \dot{\theta}_{A} \\
& =\left(\tau_{\text {loss }}-\tau_{g A}\right) \dot{\theta}_{A} \\
P_{m, B} & =\left(\tau_{B}-\tau_{b f B}-I_{g A} \ddot{\theta}_{B}\right) \dot{\theta}_{B} \\
& =\left(\tau_{\text {loss } B}-\tau_{g B}\right) \dot{\theta}_{B}
\end{aligned}
$$

The total mesh loss is:

$$
P_{\text {loss }}=P_{\text {lossA }}+P_{\text {loss } B}
$$

To distribute the losses over both gear wheels the following assumption is made:

Assumption 2 The mesh power losses are equally distributed over both gear wheels.

This assumption leads to:

$$
\tau_{\text {loss } A} \omega_{A}=\frac{P_{\text {loss }}}{2}=\tau_{\text {loss } B} \omega_{B}
$$

Using the sign conventions from Figure 3 this leads to the conclusion that a power flow into the gearbox is positive. Therefore the power loss is defined as:

$$
P_{l o s s}=P_{m A}+P_{m B}
$$

Gearbox efficiency $\eta$ depends on the power flow through the gear ${ }^{1}$. Using operational quadrants (see also Table 2), the efficiency in each quadrant is defined by following definitions:

Definition 1 The efficiency of the gearbox in quadrant 1 and 3 is:

$$
\eta=-\frac{P_{m B}}{P_{m A}}=\eta_{1}
$$

Definition 2 The efficiency of the gearbox in quadrant 2 and 4 is:

$$
\eta=-\frac{P_{m A}}{P_{m B}}=\eta_{2}
$$

\footnotetext{
${ }^{1} \mathrm{~A}$ good example is a worm wheel drive. In such a drive the efficiency from worm to gearwheel is usually $>0.5$. However, the efficiency from gearwheel to worm is in some cases zero.
}

Combining Assumption 2 with Definitions 1 and 2, the friction moment on the axis for quadrant 1 and 3 is for $\dot{\theta} \neq 0$ :

$$
\begin{aligned}
\tau_{\text {lossA }} & =-\frac{1-\eta_{1}}{1+\eta_{1}} \tau_{g A} \\
\tau_{\text {loss } B} & =-\frac{1-\frac{1}{\eta_{1}}}{1+\frac{1}{\eta_{1}}} \tau_{g B}
\end{aligned}
$$

For quadrant 2 and 4 the power loss and friction moment on the axis is:

$$
\begin{aligned}
\tau_{\text {loss } A} & =-\frac{1-\frac{1}{\eta_{2}}}{1+\frac{1}{\eta_{2}}} \tau_{g A} \\
\tau_{\text {loss } B} & =-\frac{1-\eta_{2}}{1+\eta_{2}} \tau_{g B}
\end{aligned}
$$

For $v_{\text {mesh }}=0$ (gear mesh can get stuck), the loss moment working on the gear wheel is set to zero, since the position where the loss is generated is fixed. This leads to:

$$
\tau_{\text {lossA }}=0, \quad \tau_{\text {loss }}=0
$$

\subsection{State Switching}

In order to define in which quadrant the gearbox is operating or if the gearbox is stuck, a state machine is developed. It switches based on the mesh velocity $v_{\text {mesh }}$, the sum of the total mesh loss forces and the force difference over the mesh.

The total mesh loss force depends on the operational quadrant. Since for $v_{\text {mesh }}=0$, it is unknown if the gearbox is operating in quadrant 1 or 3 (motor mode) or in quadrant 2 or 4 (generator mode). As the mesh forces $\left(F_{g A}\right.$ and $\left.F_{g B}\right)$ are known it is possible to develop a maximum and minimum loss torque for each hull. The quadrants 1 and 2 lead to $\tau_{\text {loss, } \max }$ using quadrant 1 for $F_{h}>0$ and quadrant 2 for $F_{h}<0$. The quadrants 3 and $4 \tau_{\text {loss,min }}$ using quadrant 4 for $F_{h}>0$ and quadrant 3 for $F_{h}<0$. This leads to the total loss forces:

$$
\begin{aligned}
& F_{\text {loss }, \text { max }}=\frac{\tau_{\text {loss }, \max A}}{r_{g A}}+\frac{\tau_{\text {loss }, \max B}}{r_{g B}} \\
& F_{\text {loss }, \min }=\frac{\tau_{\text {loss }, \min A}}{r_{g A}}+\frac{\tau_{\text {loss } \min B}}{r_{g B}}
\end{aligned}
$$

The force difference over the mesh is:

$$
\Delta F_{g}=-\left(\frac{\tau_{g A}}{r_{g A}}+\frac{\tau_{g B}}{r_{g B}}\right)=F_{g B}-F_{g A}
$$

Figure 4 shows how the mode switching takes place. 


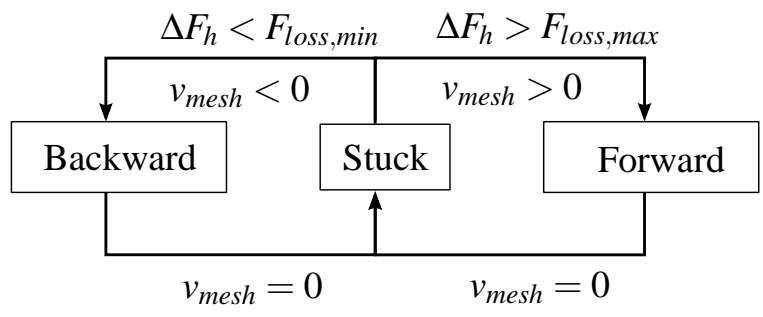

Figure 4: Mode switching of the elastic gearbox

\subsection{Mesh Stiffness- and Damping Variations}

Kar and Mohanty [3], Li et al. [4] as well as Kahraman and Singh [2] report that internal gearbox vibrations are caused by the variation of the mesh stiffness between two adjacent teeth in contact. Nevzat et al. [5] report that also damping is an important factor in gear vibrations. Moreover Li et al. [4] note that gear root cracks lead to a lower local stiffness.

To model these variations, a normalized gear stiffnesand damping profile $\left(\Delta k_{h}, \Delta c_{h}\right)$ is introduced for each gearwheel that specifies the local stiffness and damping over the circumference of each gear wheel. Using this profile the stiffness and damping of the contact point is calculated using:

$$
\begin{aligned}
& k_{h}=k_{h, \text { base }} \cdot \Delta k_{h A}\left(\theta_{A}\right) \cdot \Delta k_{h B}\left(\theta_{B}\right) \\
& c_{h}=c_{h, \text { base }} \cdot \Delta c_{h A}\left(\theta_{A}\right) \cdot \Delta c_{h B}\left(\theta_{B}\right)
\end{aligned}
$$

In Figure 5 an example of the two normalized stiffness profiles and the total local stiffness is given for two gear ratios $i=1$ and $i=2$.

\subsection{Bearing Losses}

In the elastic lossy gear model it is possible to have a stuck mesh and at the same time moving gearwheels. Therefore the mesh- and bearing losses cannot be lumped like Pelchen et al. [7] do. Instead two bearings are modelled, one on each gear wheel side. Since usually the bearings from gearboxes are not identical, each bearing can have individual friction characteristics. The same approach as Otter et al. [6] is used to model the bearing friction (this is in fact the bearing friction model from the Modelica Standard Library).
Stiffness profiles of gearwheel $A$ and $B$

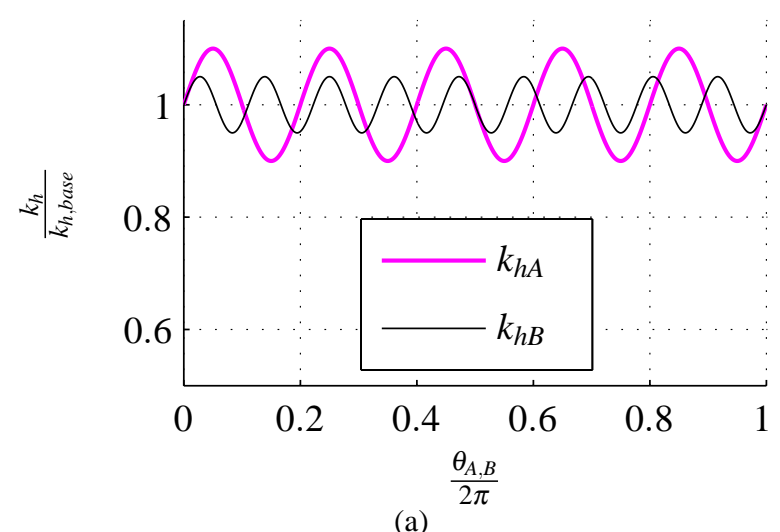

Local gear stiffness

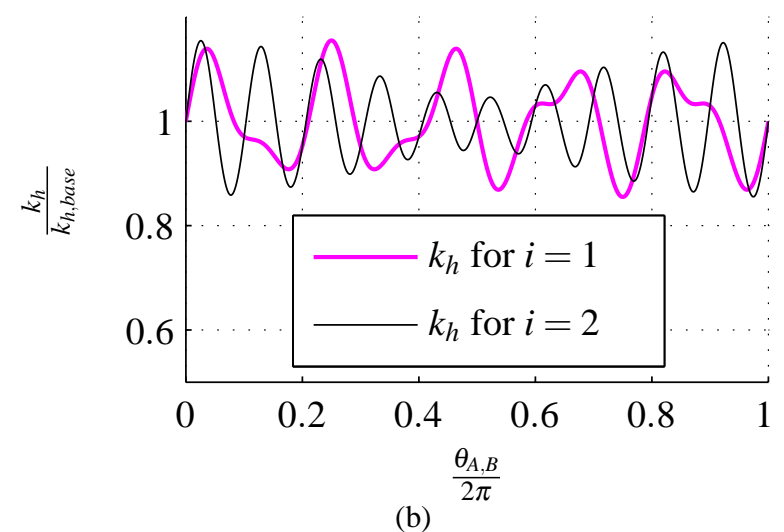

Figure 5: Local stiffness of two contacting gear wheels for gear ratios $i=1$ and $i=2$

\section{Modelica Model}

The elastic lossy gear model as developed in Section 3 can be implemented straightforward into a Modelica model. The parameters for the simulation of a 1-DOF model are the gear wheel radii $r_{A}$ and $r_{B}$, the moments of inertia of the gearwheels $I_{g A}$ and $I_{g B}$ and the nominal stiffness $k_{h}$ and damping $c_{h}$ of the mesh. Tabulated values of $\eta_{n 1}, \eta_{n 2}$ as a function of $v_{\text {mesh }}, \tau_{b f A}$ and $\tau_{b f B}$ have to be given as a function of $\dot{\theta}_{A}$ respectively $\dot{\theta}_{B}$. The last inputs are the profile tables $\Delta k_{h A}, \Delta k_{h B}, \Delta c_{h A}$ and $\Delta c_{h B}$ which are a function of the normalized circumference of the gear wheel.

\section{Simulation Results}

To check if the simulation results of the elastic lossy gear model correspond with the simulation results of the standard lossy gear model (extended with a dummy mass and two spring and damper elements) a simulation is executed with both models. A schematic 


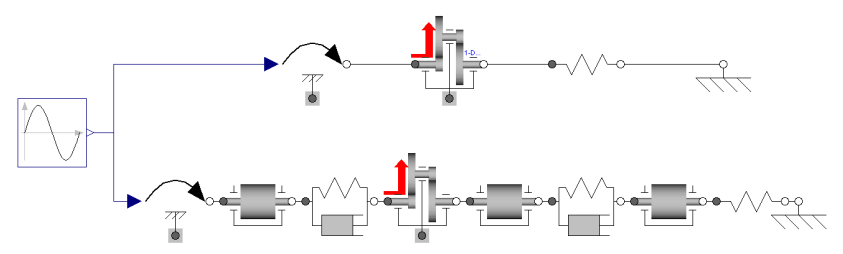

Figure 6: Elastic 1-DOF lossy gear model and standard lossy gear model extended with dummy mass and two spring- damper combinations.

overview of the models can be found in Figure 6. Both gears are driven by a sinusoidal torque (left). The loading of the gearbox takes place by a spring (right) with very low stiffness $(1 \mathrm{~N} / \mathrm{m})$. Note that this dummy mass is a work-around to avoid simulation problems. It introduces higher dynamics and moreover increases the simulation order. For a smooth simulation, the standard "Dassl" integrator requires the dummy mass to be maximal $10^{6}$ times smaller than the main masses. Implementing a 3-DOF system using the Modelica Standard Library is hardly possible and therefore not worked out.

\subsection{Gearbox Sticking}

The simulation results of the gearbox getting stuck, are shown in Figure $7^{2}$. This figure demonstrates that the simulation results are almost identical for both torsional models, leading to the conclusion that the 1-DOF elastic lossy gear model delivers the right results. The simulation results also show that the eigenfreuquency of the 3-DOF model is lower than the 1DOF model. This can be explained by the lower stiffness and damping of the 3-DOF model than the 1DOF model, caused by the extra spring-damper combination at the bearings. Note that the bearing stiffness is set to a low value to make the differences extra clear.

In addition the simulation results show clearly that in the case of a blocked gearbox, both sides of the gearbox are uncoupled; the eigenfrequency of wheel $A$ is higher than of wheel $B$. This seems not logical at first sight, since the inertia of wheel $A$ is higher than of wheel $B$. Yet the stiffness of a gearbox has a quadratic relation with the gear ratio, leading to a 9 times higher stiffness of wheel $A$ with respect to wheel $B$. Since the inertia of wheel $A$ is only 4 times higher than of wheel

\footnotetext{
${ }^{2}$ The simulation parameters are: $k_{h}=1 e 6 \mathrm{Nm}^{-1}, c_{h}=$ $10 \mathrm{Nsm}^{-1}, \eta_{1}=\eta 2=0.5, I_{g A}=4 e-3 \mathrm{kgm}^{2}, I_{g B}=1 e-3 \mathrm{kgm}^{2}$, $r_{g A}=0.3 m$ and $r_{g B}=0.1 m$.
}

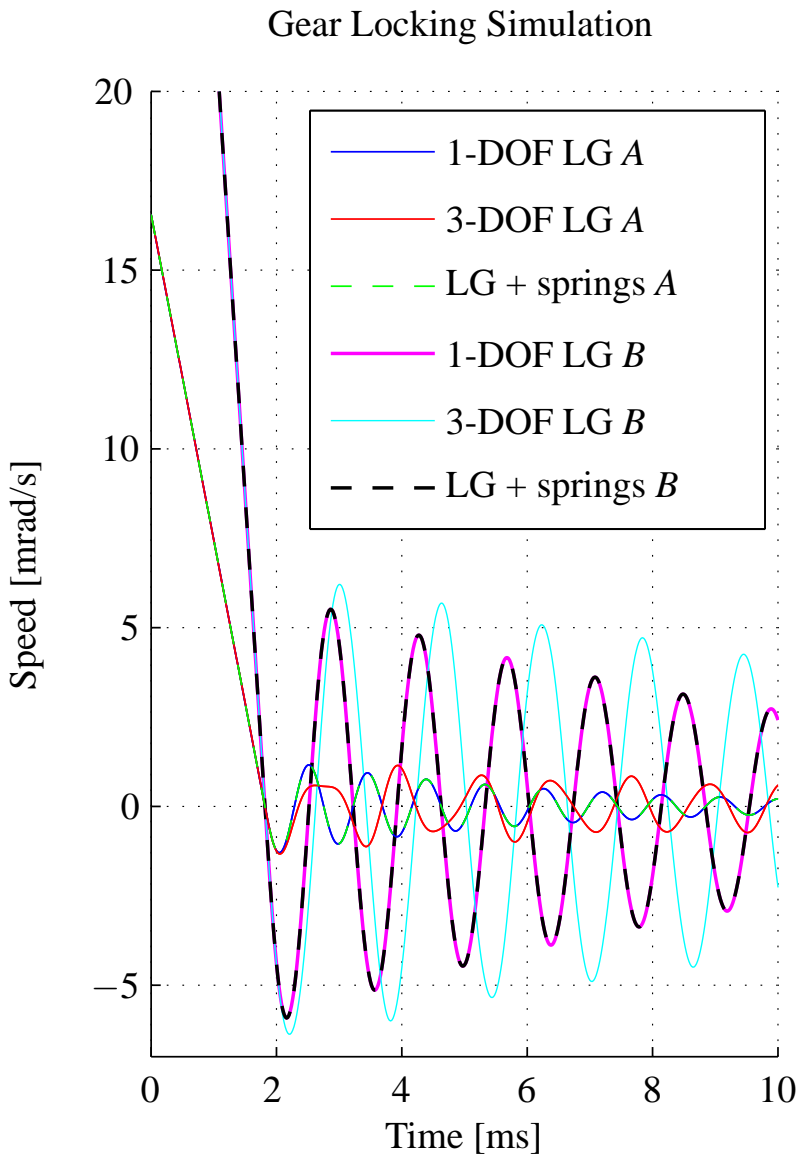

Figure 7: Simulation of the elastic 1-DOF and 3-DOF lossy gear model together with the standard lossy gear model extended with dummy mass and springs.

$B$, the eigenfrequency of wheel $A$ is $\sqrt{\frac{k}{m}}=\sqrt{\frac{9}{4}}=1.5$ times higher than wheel $B$.

\subsection{Internal Gearbox Vibrations}

As concluded in Section 3.3 the stiffness variation of the gear mesh between the gear teeth is an important source of gearbox vibrations. To simulate this behaviour a gearbox is modelled using realistic parameters for radii, stiffness and damping. The mesh stiffness variation is modelled by using the stiffness profile from Section 3.3. To simulate two gear wheels with 60 teeth, both gearwheels are given a stiffness variation profile $\left(\Delta k_{h A}\right.$ and $\left.\Delta k_{h B}\right)$ with a sinusoidal variation with 60 periods and an amplitude of $2.5 \%$ of $k_{h \text {,base }}$. The average of the profile is one. Combining both gear wheels lead to a $10 \%$ fluctuation of the gear stiffness varying 60 times each rotation.

The simulation setup is shown in Figure 8. In this figure the gear is driven by a constant speed block (left) and loaded by a constant load block (right). The elas- 


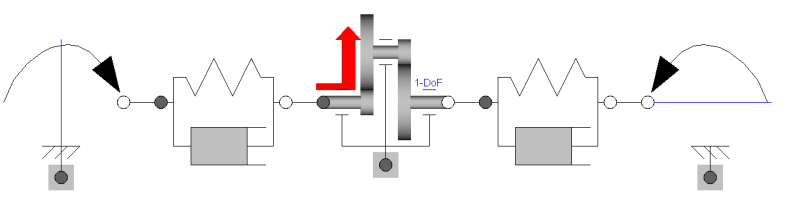

Figure 8: Simulation environment for the internal gear vibration test.

Rotational velocity of gear wheel $B$

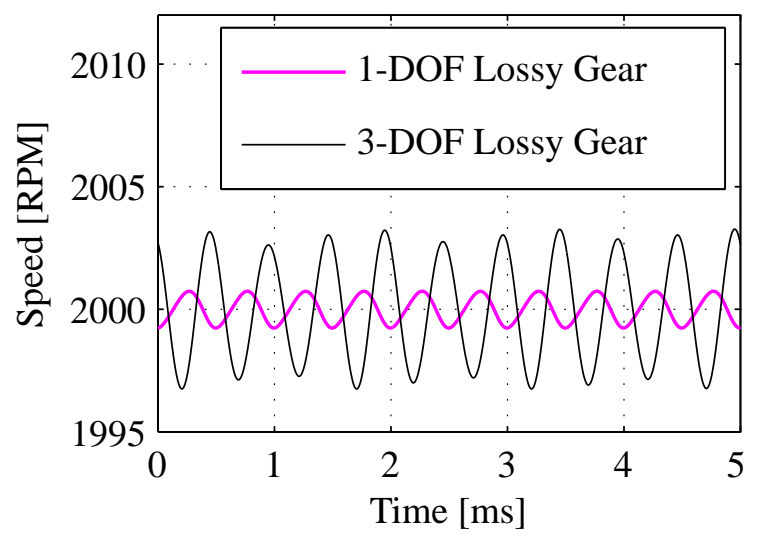

(a)

Mesh force $F_{h A}[\mathrm{~N}]$

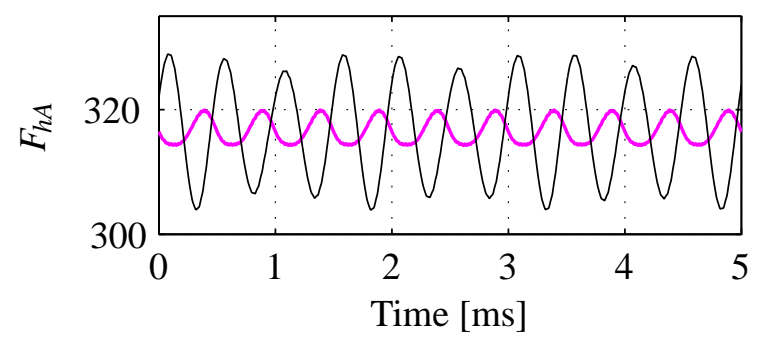

(b)

Figure 9: Simulation of the Elastic lossy gear model with a varying tooth stiffness at 2000 RPM:

tic gearbox is coupled using two relatively stiff couplings of $20 \mathrm{kNM} / \mathrm{rad}$ to a constant speed and a constant torque block. The simulation results for the gear running stationary at 2000 RPM are illustrated in Figure $9^{3}$. The extra elasticity of the bearings lowers the eigenfrequency of the vibrations (2000 RPM is close to the eigenfrequency of the 3-DOF lossy gear model). Just like in Section 5.1, the bearing stiffness and damping is chosen relatively low to show the effect of bearing stiffness.

\footnotetext{
${ }^{3} k_{h}=1 e 8 \mathrm{Nm}^{-1}, c_{h}=50 \mathrm{Nsm}^{-1}, \eta_{a}=\eta_{2}=0.9, I_{g A}=9 e-$ $5 \mathrm{kgm}^{2}, I_{g B}=9 e-5 \mathrm{kgm}^{2}, r_{g A}=30 \mathrm{~mm}$ and $r_{g B}=30 \mathrm{~mm}$.

The extra parameters for the 3-DOF model are:

$m_{A}=0.3 \mathrm{~kg}, m_{A}=0.5 \mathrm{~kg}, k_{A}=k_{B}=1 e 8 \mathrm{Nm}^{-1}, c_{A}=c_{B}=5 \mathrm{Nsm}^{-1}$
}

\section{Conclusion}

The lossy gear model of the Modelica Standard Library is extended with two models; a 1-DOF model, simulating tooth stiffness and a 3-DOF model, simulating tooth and bearing stiffness. Elasticity of the gearbox is dealt with in an appropriate way without the need for dummy masses. Just like the standard lossy gear model, chattering is avoided in this model by the state switching algorithm.

With the 1-DOF and 3-DOF elastic lossy gear models it is now possible to model the torsional as well as the translational (in load direction) vibrations of gearboxes. In addition it is possible to simulate the change of stiffness and/ or damping between the two adjacent teeth of a gear. This facilitates the modelling of vibrations that are internally generated. Furthermore, the 3-DOF model can simulate the effect of elastic bearings. The extra elasticity caused by the bearings will decrease the lowest eigenfrequency, which can cause huge problems in high velocity gear applications. The possibility to easily simulate these problems makes it possible to identify problems in an early design stage. 


\section{Appendices}

\section{A Lossy Gear Simulation Problems}

The lossy gear model from the Modelica Standard Library can not simulate when it is directly coupled with two springs. In this appendix an example will be used to demonstrate where the simulation problems originate.

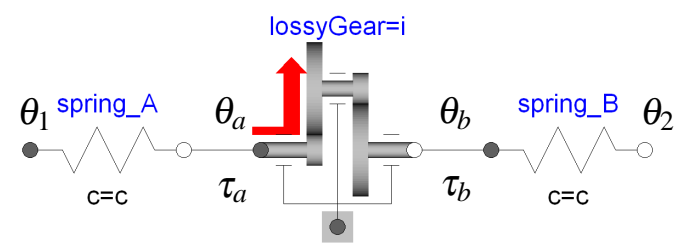

Figure 10: Lossy gear model extended with two springs

The equations of motion for the model in Figure 10 using gear ratio $i=1$ are shown in equation 37 to 40 :

$$
\begin{gathered}
\theta_{a}=i \theta_{b}=\theta_{b} \\
\tau_{a}=c\left(\theta_{a}-\theta_{1}\right) \\
\tau_{b}=c\left(\theta_{2}-\theta_{b}\right) \\
\tau_{\text {loss }}= \begin{cases}\text { stuck } & :-\left(\tau_{a}+\tau_{b}\right) \text { so that } \ddot{\theta}_{a}=0 \\
\text { sliding } & : k\left(\dot{\theta}_{a}\right) \dot{\theta}_{a}\end{cases}
\end{gathered}
$$

In Equation $40, k\left(\dot{\theta}_{a}\right)$ is a variable defining the efficiency of the lossy gear model (which can be dependant on $\dot{\theta}_{a}$ ).

Combining equations 37 to 40 yield the following differential equations for the stuck mode as well as for the sliding mode:

$$
\begin{array}{cl}
\text { stuck: } & c\left(\theta_{a}-\theta_{1}\right)+c\left(\theta_{2}-\theta_{a}\right)+k\left(\dot{\theta}_{a}\right)=0 \\
\text { sliding: } & \begin{cases}\tau_{\text {loss }}=-\left(c\left(\theta_{a}-\theta_{1}\right)+c\left(\theta_{2}-\theta_{a}\right)\right) \\
\ddot{\theta}_{a}=0\end{cases}
\end{array}
$$

Comparing Equation 41 and 42 shows that the equation in stuck mode (Eq 41) is a differential equation of first order in $\theta_{a}\left(\theta_{1}\right.$ and $\theta_{2}$ are input signals to this equation). On the contrary, the differential equation for sliding (Eq 42) is of second order in $\theta_{a}$.

Summing up, this leads to a changing number of differential equations while switching between stuck and sliding mode. Dymola (and also other Modelica tools) cannot handle cases in which the number of differential equations changes during simulation. Therefore the model as shown in Figure 10 cannot simulate any switching between stuck and sliding.
A method to fix this problem would be to replace $\ddot{\theta}_{a}=0$ from Equation 42 by $\dot{\theta}_{a}=0$, yielding a model that does not change states. However this is non-trivial because all switching conditions (how to switch between sliding and stuck mode) would change.

\section{References}

[1] I. Howard, S. Jia, and J. Wang. The dynamic modelling of a spur gear in mesh including friction and a crack. Mechanical Systems and Signal Processing, 15:831-853, 2001.

[2] A. Kahraman and R. Singh. Interactions between time-varying mesh stiffness and clearance nonlinearities in a geared system. Journal of Sound and Vibration, 142:49-75, 1990.

[3] C. Kar and A. Mohanty. Determination of timevarying contact length, friction force, torque and forces at the bearings in a helical gear system. Journal of Sound and Vibration, 309:307-319, 2008.

[4] C. J. Li, H. Lee, and S. H. Choi. Estimating size of gear tooth root crack using embedded modelling. Mechanical Systems and Signal Processing, 16:841-852, 2002.

[5] H. Nevzat, Özgüven, and D. Houser. Dynamic analysis of high speed gear by using loaded static transmission error. Journal of Sound and Vibration, 125:71-83, 1988.

[6] M. Otter, H. Elmqvist, and S. E. Mattsson. Hybrid modeling in modelica based on the synchronous data flow principle. In $C A C S D$, Hawaii, USA, August 1999.

[7] C. Pelchen, C. Schweiger, and M. Otter. Modeling and simulating the efficiency of gearboxes and of planetary gearboxes. In 2nd International Modelica Conference, pages 257-266, 2002.

[8] R. Sing and D. R. Houser. Non-Linear Dynamic Analysis of Geared Systems. $\mathrm{PhD}$ thesis, Ohio State University, February 1990. 